\title{
Potato thin layer convective dehydration model and energy efficiency estimation
}

\author{
Marko Petković1,*, Alexander Lukyanov ${ }^{2}$, Dmitry Rudoy ${ }^{2}$, Vladimir Kurćubić1 ${ }^{1}$ Igor \\ Đurović ${ }^{1}$, Nemanja Miletić ${ }^{1}$, and Jasur Safarov ${ }^{4}$ \\ ${ }^{1}$ University of Kragujevac, Faculty of Agronomy, Department of Food Technology, Cara Dušana 34, \\ Čačak, Serbia \\ ${ }^{2}$ Don State Technical University, square Gagarin 1, Rostov on Don, 344003 Russian Federation \\ ${ }^{3}$ Tashkent state technical University named after Islam Karimov, 100095, 2, str. University Tashkent, \\ Uzbekistan
}

\begin{abstract}
The dehydration parameters (temperature, thickness, and mass load) statistically significantly $(\mathrm{p}<0.05)$ affect the thin-layer convective dehydration of potato slices. The slices with thicknesses of 3, 5, and $8 \mathrm{~mm}$ were dehydrated as monolayers at different temperatures $\left(30,50\right.$, and $\left.70^{\circ} \mathrm{C}\right)$ and mass load $(1.00,0.63$, and $0.38 \mathrm{~kg} \mathrm{~m}-2)$. The results showed that the shortest dehydration time (183 minutes), the smallest energy consumption $(0.176 \mathrm{kWh})$, and the smallest emission of carbon dioxide $(0.17 \mathrm{~kg})$ had the dehydration model of potato slices with a $3 \mathrm{~mm}$ thickness, $0.38 \mathrm{~kg} \mathrm{~m}-2$ mass load, dehydrated on the temperature of $70^{\circ} \mathrm{C}$. Dehydration of potato slices of $8 \mathrm{~mm}$ slice thickness dehydrated at $70{ }^{\circ} \mathrm{C}$, with $0.38 \mathrm{~kg} \mathrm{~m}-2$ mass load, showed the highest resistance to mass transfer (the maximum effective moisture diffusivity $2.3761 \times 10-7 \pm 4.45646 \times 10-9 \mathrm{~m} 2 \mathrm{~s}-1)$ and the minimum activation energy $(27.02 \mathrm{~kJ}$ mol-1). Data obtained from these mathematical models could predict and optimize the thin layer dehydration of potato slices, with a dominant influence of temperature and potato slice thickness parameters as variables.
\end{abstract}

\section{Introduction}

Due to the high moisture content, safe storage of freshly harvested agricultural products is challenging, and the materials' quality is rapidly lost. Dehydration is the primary physical method to ensure safe and long storage and usage diversity of the foodstuffs [1]. Among the different dehydration models, convective hot air dehydrators are the widely used systems to dehydrate agricultural and food products [2]. The traditional convective dehydration has limited heat transfer from the hot air into the dehydration material's inner sections, resulting in non-efficient consumption of energy, contamination of the environment, a low dehydration time, and low quality of dehydrated products [3, 4].

Dehydration is defined as removing liquid from a solid by the thermal application [5]. The factors that affect the dehydration efficiency depend on the dehydrating medium (temperature, hot air speed, air humidity, power supply, etc.) and materials to be dehydrated

\footnotetext{
${ }^{*}$ Corresponding author: marko.petkovic@kg.ac.rs
} 
(size, thickness, mass load, structure, initial moisture content, and the partial pressure in the material [6]. Convective dehydration is one of the oldest technologies for preserving fresh agricultural products, food, and herbs. Generally, the primary purpose of dehydration is to reduce the moisture content, allowing the dehydrated products to be stored for a longer period (low microorganisms and enzymes' activity) and to minimize the transport costs and storage $[7,8,9]$.

Dehydration involves heat and mass transfer mechanisms. Hence, an understanding of the food and agriculture crops' engineering properties will help design dehydrators and optimize the process parameters and energy consumption systems. They are essential in the quantitative evaluation of energy requirements and energy losses in drying systems [10]. Thin-layer dehydration refers to the drying process. All the products were fully exposed to the hot air under constant dehydration parameters, i.e., constant air temperature, speed, and humidity. The temperature has the most substantial impact on losing essential substances in a material. The higher the dehydration material's temperature, the faster the raw material dried up to a constant weight [11].

Therefore, this study's primary objectives were to investigate the models of convective thin-layer dehydration of potato slices and assess the influence of dehydration parameters (dehydration temperature, potato slice thickness, and a mass load) on moisture removal features of the potato slices, energy consumption, and the effective moisture diffusivity and activation energy of the process. Dehydrated potato slices are a semi-finished product, which can be further fried to obtain a snack product or ground to obtain potato flour.

\section{Materials and Methods}

\subsection{Potato thin layer convective dehydration}

Fresh white potatoes (Kennebec variety) were harvested from a Paraćin area $\left(43^{\circ} 51^{\prime} 46.9^{\prime \prime} \mathrm{N}\right.$ $21^{\circ} 24^{\prime} 19.7^{\prime \prime} \mathrm{E}$, Serbia), and stored in a refrigerator at $4{ }^{\circ} \mathrm{C}$. The fresh potatoes' initial moisture content was obtained to be $6.17 \pm 0.21 \mathrm{~kg}$ water $\mathrm{kg}-1$ dry matter [12]. Before each dehydration experiment, the fresh potatoes were taken out of the refrigerator, washed with water, placed at the ambient temperature for a couple of hours to stabilize, peeled, and cut to the desired thicknesses. Convective dehydrator (Colossus CSS 5330 250W, PRC) was used to dry the potato slices at temperatures of 35,50 , and $70{ }^{\circ} \mathrm{C}$ at atmospheric pressure, to the constant weight. Potato slices were uniformly distributed as a thin layer on the $320-\mathrm{mm}$ diameter tray with a mass load of $0.38,0.63$, and $1.00 \mathrm{~kg} \mathrm{~m}-2$. The convective dehydration was operated at an air velocity of $3.0 \mathrm{~m} \mathrm{~s}^{-1}$ [13]. The convective drying kinetic was based on mass losses of potato slices $[4,14]$.

\subsection{Mathematical model of dehydration}

The moisture ratio (MR) is defined according to Eq. (1):

$$
M R=\frac{M_{t}-M_{e}}{M_{o}-M_{e}}
$$

Mt, Mo, and Me represent the moisture content achieved after convective drying time t, the initial moisture content, and the equilibrium moisture content, respectively. The value of equilibrium moisture content (Me) is usually deficient and can be deleted from Eq. (1) without a significant change of MR. 


\subsection{Determination of effective moisture diffusivity $\left(D_{\text {eff }}\right)$}

The most widely used theoretical models to describe the internal diffusion phenomenon on dehydration of vegetables and fruits were derived from Fick's second law of diffusion. The analytical solutions according to the product geometry (slices, Eq. (1) and (2)) were given below [15]:

$$
\begin{gathered}
\mathrm{MR}=\mathrm{A}_{1} \cdot \sum_{\mathrm{i}=1}^{\alpha} \frac{1}{\mathrm{~J}_{\mathrm{o}}^{2}} \cdot e^{-\frac{\mathrm{J}_{0}^{2} \cdot \mathrm{D}_{\text {eff }}}{\mathrm{A}_{2}}} \\
\mathrm{~A}_{1}=\frac{8}{\pi^{2}} ; \quad \mathrm{A}_{2}=4 \cdot \mathrm{L}^{2}
\end{gathered}
$$

Where Deff is the effective moisture diffusivity ( $\mathrm{m} 2 \mathrm{~s}-1)$, $\mathrm{t}$ is time (s), MR is the moisture ratio, $\mathrm{J} 0$ is the roots of the Bessel function, A1, A2 are geometric constants, and L is the thickness of the potato slice if dehydration has occurred through only one side.

Eq. 1 is derived for the constant values of Deff and for sufficiently long drying time:

$$
\begin{gathered}
\ln (M R)=\ln (a)-k \quad t \\
k=-\frac{\pi^{2} \cdot D_{\text {eff }}}{A_{2}}
\end{gathered}
$$

The variation of $\ln (\mathrm{MR})$ versus $t$ is linear (Eq. (4)), and the slope is equal to the drying constant $(\mathrm{k})$, and the constant effective moisture diffusivity can be easily calculated (Eq. (5) $[15,16]$.

\subsection{Estimation of activation energy (Ea)}

The activation energy (Ea) is a measure of the necessary energy required to begin the water diffusion from the dehydration material's internal areas. Generally, high activation energy values are related to materials in which water is more strongly bound to the structure, and the samples' structure carries out water removal [3]. The activation energy was calculated using an Arrhenius type equation (Eq. (6)) [16]:

$$
\mathrm{D}_{\text {eff }}=\mathrm{D}_{0} \cdot \mathrm{e}^{-\frac{\mathrm{E}_{\mathrm{a}}}{\mathrm{R} \cdot \mathrm{T}}}
$$

Where Ea is the activation energy ( $\mathrm{kJ}$ mol-1), $\mathrm{R}$ is the universal gas constant $(8.3143 \mathrm{~J}$ mol-1 K-1), $\mathrm{T}$ is absolute air temperature $(\mathrm{K})$, and D0 is the pre-exponential factor of the Arrhenius equation (m2 s-1). The activation energy was calculated from the slope of the Arrhenius plot $\ln (\mathrm{De})$ versus $1 / \mathrm{T}$, given by Eq. (7):

$$
n=-\frac{E_{a}}{R}
$$

\subsection{Energy consumption (E)}

Energy consumption (E) for the dehydration of potato slices was measured by Prosto PM 001 $\left(230 \mathrm{~V}, 50 \mathrm{~Hz}, 0-16 \mathrm{~A}, 2-3680 \mathrm{~W}, 0-9999 \mathrm{kWh},-10{ }^{\circ} \mathrm{C}\right.$ to $+40{ }^{\circ} \mathrm{C}, \leq 85 \%$ of relative humidity, the altitude of use max $2000 \mathrm{~m}$ ). This two-tariff electricity consumption meter also can calculate the price of consumed energy, so it is instrumental in planning the implementation of electricity-saving measures. The amount of consumed energy is mathematically correlated with the emitted carbon dioxide $(1 \mathrm{kWh}$ releases $0.998 \mathrm{~kg} \mathrm{CO} 2)$ [17]. 


\subsection{Statistical analysis}

Response Surface Methodology (RSM) and Analysis of variance (ANOVA) were selected to estimate the dehydration variables of potato slices (dehydration temperature, thickness, and mass load). The independent variables were dehydration temperature of 35,50 , and $70{ }^{\circ} \mathrm{C}$, potato slice thickness $(3,5$, and $8 \mathrm{~mm})$ and mass load $(1.00,0.63$, and $0.38 \mathrm{~kg} \mathrm{~m}-2)$ for dehydration (X1-3). The dependent variables were dehydration time ( $t$, Y1-9), energy consumption (E, Y10-18), and coefficient of diffusion (Deff, Y19-27). The emission of CO2 was not statistically analyzed because of the mathematical correlation estimated by the Prosto manufacturer $(1 \mathrm{kWh}$ releases $0.998 \mathrm{~kg} \mathrm{CO} 2)$. The models were fitted to the response surface generated by the experiment. The following second-order polynomial (SOP) model was fitted to the data:

$$
Y_{k}=\beta_{k 0}+\sum_{i=1}^{2} \beta_{k i} X_{i}+\sum_{i=1}^{2} \beta_{k i l} X_{i}^{2}+\beta_{k i j} X_{i} X_{j}, \mathrm{k}=1-27
$$

Where $\beta$ kij are constant regression coefficients. The evaluations of ANOVA (Tukey HSD Test) analyses were performed using STATISTICA 12.0 software (2013) [18].

\section{Results}

The average dehydration time (the time when the dehydrated mass was constant), energy consumption, the emission of carbon dioxide (CO2), and the effective moisture diffusivity of thin-layer dehydration of potato slices, based on the different mass load $(1.00,0.63$, and 0.38 $\mathrm{kg} \mathrm{m}-2$ ), are presented in Tables $1-3$, respectively. All experimental results were analyzed in triplicates.

In Table 4, the average values of activation energy are presented. The results are shown based on the different mass load $\left(1.00,0.63\right.$, and $\left.0.38 \mathrm{~kg} \mathrm{~m}^{-2}\right)$. The average values and standard deviations of activation energy at different drying temperatures $\left(35,50,70{ }^{\circ} \mathrm{C}\right)$ and thickness $(3,5,8 \mathrm{~mm})$ of potato slices are shown in Figure 1.

Table 5 presents the results of Anova analysis of response surface models (RSM) based on experimental data for the dehydration time, energy consumption, the effective moisture diffusivity, and the activation energy. The statistical significance of all models and residual variances is noted, with the determination coefficient (R2). Regression coefficients of all SOP models for the dehydration time, energy consumption, the effective moisture diffusivity, and the activation energy are shown in Tables 6 . The statistical significance of individual coefficients was noted. Data obtained from these mathematical models could predict and optimize the thin layer dehydration of potato slices based on the experimental plan. These parameters for the emission of carbon dioxide $(\mathrm{CO} 2)$ are equal to the energy consumption parameters.

Table 6 presents the regression coefficients of SOP of dehydration time, energy consumption, the effective moisture diffusivity, and activation energy of potato slices dehydration.

\section{Discussion}

The initial and final moisture contents of the material are the main factor controlling the dehydration time and, of course, all the other experimental parameters, as well as the dehydration system and inherent properties of the material (tissue structure, the material form such as a sphere, infinite slab, the hot air flow direction, etc.) [15].

Based on the experimental results (Tables $1-3$ ), the required time to dehydrate the potato slices statistically significantly $(\mathrm{p}<0.05)$ depends on process parameters (dehydration 
temperature, the thickness of potato slices, and mass load fo the dehydration process). At the same mass load, increasing the dehydration temperature, the dehydration time ( $t$ ) was decreased; the average kinetic energy of the moisture was increased, and the diffusion process of moisture was easier. Unlike temperature, with increasing the thickness of potato slices, the dehydration time was increased because of the drying rate reduction (the increased distance the moisture travels in material with large thickness) [19]. Similar results on the effect of material thickness on dehydration time and rate have been reported for sweet potato [20]. As far as the mass load was decreased, the dehydration time was decreased too. The shortest dehydration time (183 minutes) was the parameter of dehydration of potato slices with the 3 mm thickness, $0.38 \mathrm{~kg} \mathrm{~m}-2$ mass load, dehydrated on temperature $70{ }^{\circ} \mathrm{C}$. In comparison, the longest dehydration time (1614 minutes) was taken to dehydrate the potato slices with the 8 $\mathrm{mm}$ thickness, $1.00 \mathrm{~kg} \mathrm{~m}-2$ mass load, dehydrated at temperature $35^{\circ} \mathrm{C}$.

Table 1. Thin layer dehydration results of potato slices (mass load $1.00 \mathrm{~kg} \mathrm{~m}^{-2}$ ).

\begin{tabular}{|c|c|c|c|c|c|c|c|}
\hline $\mathbf{T}\left({ }^{\circ} \mathrm{C}\right)$ & $\begin{array}{c}\mathbf{m} \\
(\mathbf{k g} \\
\left.\mathbf{m}^{-2}\right)\end{array}$ & $\begin{array}{c}\text { d } \\
(\mathbf{m m})\end{array}$ & $\begin{array}{c}\mathrm{t} \\
(\mathrm{min})\end{array}$ & $\begin{array}{c}\mathbf{E} \\
(\mathbf{k W h})\end{array}$ & $\begin{array}{c}\mathbf{k g} \\
\left(\mathrm{CO}_{2}\right)\end{array}$ & $\underset{\mathbf{E}}{\mathrm{CO}_{2} /}$ & $\underset{\left(\mathrm{m}^{2} \mathrm{~s}^{-1}\right)}{D_{\text {eff }}}$ \\
\hline \multirow{3}{*}{35} & \multirow{9}{*}{1.00} & 3 & $\begin{array}{c}695 \pm \\
6^{14} \\
\end{array}$ & $\begin{array}{l}0.814 \pm \\
0.007^{3,4}\end{array}$ & $\begin{array}{c}0.81 \pm \\
0.008^{3,4}\end{array}$ & $\begin{array}{c}0.99 \\
4\end{array}$ & $\begin{array}{l}2.3003 \times 10^{-8} \pm 2.1837 \times \\
10^{-101}\end{array}$ \\
\hline & & 5 & $\begin{array}{r}1545 \\
\pm 18^{17} \\
\end{array}$ & $\begin{array}{l}1.613 \pm \\
0.019^{11} \\
\end{array}$ & $\begin{array}{r}1.61 \pm \\
0.021^{11} \\
\end{array}$ & $\begin{array}{c}0.99 \\
7\end{array}$ & $\begin{array}{l}3.0427 \times 10^{-8} \pm 3.5375 \times \\
10^{-102,3}\end{array}$ \\
\hline & & 8 & $\begin{array}{c}1614 \\
\pm 19^{18}\end{array}$ & $\begin{array}{l}1.916 \pm \\
0.022^{13}\end{array}$ & $\begin{array}{l}1.92 \pm \\
0.022^{13}\end{array}$ & $\begin{array}{c}0.99 \\
9\end{array}$ & $\begin{array}{l}5.7122 \times 10^{-8} \pm 6.65672 \times \\
10^{-105}\end{array}$ \\
\hline \multirow{3}{*}{50} & & 3 & $\begin{array}{c}310 \pm \\
5^{5} \\
\end{array}$ & $\begin{array}{l}1.271 \pm \\
0.022^{9}\end{array}$ & $\begin{array}{l}1.27 \pm \\
0.024^{9}\end{array}$ & $\begin{array}{c}0.99 \\
6 \\
\end{array}$ & $\begin{array}{l}4.5641 \times 10^{-8} \pm 8.0403 \times \\
10^{-104}\end{array}$ \\
\hline & & 5 & $\begin{array}{l}513 \pm \\
8^{10,11} \\
12\end{array}$ & $\begin{array}{l}2.106 \pm \\
0.034^{14}\end{array}$ & $\begin{array}{l}2.10 \pm \\
0.034^{14}\end{array}$ & $\begin{array}{c}0.99 \\
8\end{array}$ & $\begin{array}{l}7.9111 \times 10^{-8} \pm 1.2627 \times \\
10^{-97}\end{array}$ \\
\hline & & 8 & $\begin{array}{c}804 \pm \\
15^{15} \\
\end{array}$ & $\begin{array}{l}3.300 \pm \\
0.062^{16}\end{array}$ & $\begin{array}{l}3.30 \pm \\
0.062^{16}\end{array}$ & $\begin{array}{c}0.99 \\
9 \\
\end{array}$ & $\begin{array}{l}1.2203 \times 10^{-7} \pm 2.3067 \times \\
10^{-9} 12\end{array}$ \\
\hline \multirow{3}{*}{70} & & 3 & $\begin{array}{c}205 \pm \\
3^{1,2} \\
\end{array}$ & $\begin{array}{l}0.877 \pm \\
0.006^{4,5} \\
\end{array}$ & $\begin{array}{c}0.87 \pm \\
0.006^{4,5} \\
\end{array}$ & $\begin{array}{c}0.99 \\
7 \\
\end{array}$ & $\begin{array}{l}6.0976 \times 10^{-8} \pm 2.55978 \times \\
10^{-105}\end{array}$ \\
\hline & & 5 & $\begin{array}{c}399 \pm \\
7^{7}\end{array}$ & $\begin{array}{l}1.711 \pm \\
0.017^{12}\end{array}$ & $\begin{array}{l}1.71 \pm \\
0.017^{12}\end{array}$ & $\begin{array}{c}0.99 \\
8\end{array}$ & $\begin{array}{l}8.6210 \times 10^{-8} \pm 5.74838 \times \\
10^{-108,9}\end{array}$ \\
\hline & & 8 & $\begin{array}{l}541 \pm \\
10^{11,12}\end{array}$ & $\begin{array}{l}2.276 \pm \\
0.018^{15}\end{array}$ & $\begin{array}{l}2.27 \pm \\
0.018^{15}\end{array}$ & $\begin{array}{c}0.99 \\
8\end{array}$ & $\begin{array}{l}1.4021 \times 10^{-7} \pm 1.04179 \times \\
10^{-9} 13\end{array}$ \\
\hline
\end{tabular}

${ }^{1-18}$ Different numbers in superscript in Table $1-3$ indicate the statistically significant difference between values, at a significance level of $p<0.05$

Table 2. Thin layer dehydration results of potato slices (mass load $0.63 \mathrm{~kg} \mathrm{~m}^{-2}$ ).

\begin{tabular}{|c|c|c|c|c|c|c|c|}
\hline $\begin{array}{c}\mathbf{T} \\
\left({ }^{\circ} \mathbf{C}\right)\end{array}$ & $\begin{array}{c}\mathbf{m} \\
(\mathbf{k g} \\
\left.\mathbf{m}^{-2}\right)\end{array}$ & $\begin{array}{l}d \\
(\mathbf{m} \\
\mathrm{m})\end{array}$ & $\underset{(\min )}{t}$ & $\begin{array}{c}\mathbf{E} \\
(\mathbf{k W h})\end{array}$ & $\begin{array}{c}\mathbf{k g} \\
\left(\mathbf{C O}_{2}\right)\end{array}$ & $\underset{\mathbf{E}}{\mathrm{CO}_{2} /}$ & $\begin{array}{c}D_{\text {eff }} \\
\left(\mathbf{m}^{2} \mathbf{s}^{-1}\right)\end{array}$ \\
\hline \multirow{3}{*}{35} & \multirow{7}{*}{0.63} & 3 & $\begin{array}{l}503 \pm \\
5^{10}\end{array}$ & $0.632 \pm 0.006^{2}$ & $0.63 \pm 0.006^{2}$ & 0.990 & $\begin{array}{l}2.5925 \times 10^{-8} \pm 2.55978 \times \\
10^{-101,2}\end{array}$ \\
\hline & & 5 & $\begin{array}{c}822 \pm \\
14^{15} \\
\end{array}$ & $\begin{array}{c}1.005 \pm 0.017^{6,} \\
7 \\
\end{array}$ & $\begin{array}{c}1.00 \pm 0.017^{6} \\
7 \\
\end{array}$ & 0.995 & $\begin{array}{l}3.4647 \times 10^{-8} \pm 5.74838 \times \\
10^{-103}\end{array}$ \\
\hline & & 8 & $\begin{array}{c}17 \\
16^{16}\end{array}$ & $\begin{array}{c}1.058 \pm 0.018^{7} \\
8\end{array}$ & $\begin{array}{l}1.06 \pm 0.018^{7} \\
8\end{array}$ & 0.991 & $\begin{array}{l}6.0122 \times 10^{-8} \pm 1.04179 \times \\
10^{-95}\end{array}$ \\
\hline \multirow{3}{*}{50} & & 3 & $\begin{array}{c}265 \pm \\
4^{4}\end{array}$ & $1.110 \pm 0.019^{8}$ & $1.11 \pm 0.019^{8}$ & 0.998 & $\begin{array}{l}4.7832 \times 10^{-8} \pm 8.10406 \times \\
10^{-104}\end{array}$ \\
\hline & & 5 & $\begin{array}{c}396 \pm \\
5^{7} \\
\end{array}$ & $\begin{array}{c}1.664 \pm \\
0.021^{11,12} \\
\end{array}$ & $\begin{array}{c}1.66 \pm \\
0.021^{11,12} \\
\end{array}$ & 0.998 & $\begin{array}{l}8.5196 \times 10^{-8} \pm 1.05744 \times \\
10^{-97,8,9}\end{array}$ \\
\hline & & 8 & $\begin{array}{c}549 \pm \\
10^{12}\end{array}$ & $\begin{array}{l}2.307 \pm \\
0.041^{15}\end{array}$ & $\begin{array}{l}2.30 \pm \\
0.041^{15}\end{array}$ & 0.999 & $\begin{array}{l}1.5838 \times 10^{-7} \pm 2.82764 \times \\
10^{-914}\end{array}$ \\
\hline 70 & & 3 & $\begin{array}{c}196 \pm \\
2^{1,2}\end{array}$ & $\begin{array}{c}0.839 \pm 0.007^{3,} \\
4\end{array}$ & $\begin{array}{c}0.82 \pm 0.007^{3} \\
4\end{array}$ & 0.974 & $\begin{array}{l}6.7914 \times 10-8 \pm 5.65164 \times \\
10^{-106}\end{array}$ \\
\hline
\end{tabular}




\begin{tabular}{|c|c|c|c|c|c|c|l|}
\hline & 5 & $\begin{array}{c}319 \pm \\
4^{5}\end{array}$ & $1.340 \pm 0.018^{9}$ & $1.34 \pm 0.018^{9}$ & 0.997 & $\begin{array}{l}1.1461 \times 10^{-7} \pm 1.51827 \times \\
10^{-9} 11\end{array}$ \\
\cline { 3 - 7 } & & $443 \pm$ & $1.862 \pm$ & $1.86 \pm$ & 0.967 & $1.6684 \times 10^{-7} \pm 2.93341 \times$ \\
& & 8 & $8^{8,9}$ & $0.033^{13}$ & $0.033^{13}$ & 0.997 & $10^{-9}$ \\
\hline
\end{tabular}

1-16 Different numbers in superscript in Table $1-3$ indicate the statistically significant difference between values, at a significance level of $p<0.05$

Table 3. Thin layer dehydration results of potato slices (mass load $0.38 \mathrm{~kg} \mathrm{~m}^{-2}$ ).

\begin{tabular}{|c|c|c|c|c|c|c|c|}
\hline $\begin{array}{c}\mathbf{T} \\
\left({ }^{\circ} \mathbf{C}\right)\end{array}$ & $\begin{array}{c}\mathbf{m} \\
(\mathrm{kg} \\
\left.\mathrm{m}^{-2}\right)\end{array}$ & $\begin{array}{l}\text { d } \\
(\mathrm{m} \\
\mathrm{m})\end{array}$ & $\begin{array}{c}\mathbf{t} \\
(\min )\end{array}$ & $\begin{array}{c}\mathbf{E} \\
(\mathrm{kWh})\end{array}$ & $\begin{array}{c}\mathbf{k g} \\
\left(\mathrm{CO}_{2}\right)\end{array}$ & $\begin{array}{c}\mathrm{CO}_{2} / \\
\mathrm{E}\end{array}$ & $\underset{\left(m^{2} s^{-1}\right)}{D_{\text {eff }}}$ \\
\hline \multirow{3}{*}{35} & \multirow{9}{*}{0.38} & 3 & $\begin{array}{c}411 \pm \\
4^{7,8} \\
\end{array}$ & $\begin{array}{c}0.176 \pm \\
0.002^{1} \\
\end{array}$ & $\begin{array}{l}0.17 \pm \\
0.002^{1}\end{array}$ & 0.982 & $\begin{array}{l}2.7750 \times 10^{-8} \pm 2.56597 \times \\
10^{-101,2}\end{array}$ \\
\hline & & 5 & $\begin{array}{l}512 \pm \\
10^{10,11} \\
\end{array}$ & $\begin{array}{c}0.217 \pm \\
0.004^{1} \\
\end{array}$ & $\begin{array}{l}0.21 \pm \\
0.004^{1}\end{array}$ & 0.985 & $\begin{array}{l}4.5641 \times 10^{-8} \pm 8.58114 \times \\
10^{-104}\end{array}$ \\
\hline & & 8 & $\begin{array}{l}598 \pm \\
12^{9,13} \\
\end{array}$ & $\begin{array}{c}0.251 \pm \\
0.005^{1} \\
\end{array}$ & $\begin{array}{l}0.25 \pm \\
0.005^{1} \\
\end{array}$ & 0.984 & $\begin{array}{l}9.3472 \times 10^{-8} \pm 1.88729 \times \\
10^{-910}\end{array}$ \\
\hline \multirow{3}{*}{50} & & 3 & $\begin{array}{c}250 \pm \\
3^{3,4} \\
\end{array}$ & $\begin{array}{l}1.048 \pm \\
0.014^{7,8} \\
\end{array}$ & $\begin{array}{c}1.05 \pm \\
0.014^{7,8}\end{array}$ & 0.998 & $\begin{array}{l}4.9292 \times 10^{-8} \pm 6.66205 \times \\
10^{-104}\end{array}$ \\
\hline & & 5 & $\begin{array}{c}355 \pm \\
4^{6}\end{array}$ & $\begin{array}{l}1.492 \pm \\
0.016^{10}\end{array}$ & $\begin{array}{c}1.49 \pm \\
0.016^{10}\end{array}$ & 0.998 & $\begin{array}{l}8.9292 \times 10^{-8} \pm 9.51758 \times \\
10^{-109,10}\end{array}$ \\
\hline & & 8 & $\begin{array}{c}455 \pm \\
5^{9} \\
\end{array}$ & $\begin{array}{l}1.911 \pm \\
0.023^{13}\end{array}$ & $\begin{array}{l}1.91 \pm \\
0.023^{13}\end{array}$ & 0.999 & $\begin{array}{l}1.6059 \times 10^{-7} \pm 1.91335 \times \\
10^{-914,15}\end{array}$ \\
\hline \multirow{3}{*}{70} & & 3 & $\begin{array}{c}183 \pm \\
1^{1} \\
\end{array}$ & $\begin{array}{c}0.764 \pm \\
0.004^{3} \\
\end{array}$ & $\begin{array}{l}0.74 \pm \\
0.004^{3}\end{array}$ & 0.971 & $\begin{array}{l}8.1788 \times 10^{-8} \pm 4.22868 \times \\
10^{-107,8}\end{array}$ \\
\hline & & 5 & $\begin{array}{c}225 \pm \\
3^{2,3} \\
\end{array}$ & $\begin{array}{l}0.942 \pm \\
0.012^{5,6} \\
\end{array}$ & $\begin{array}{c}0.94 \pm \\
0.012^{5,6} \\
\end{array}$ & 0.994 & $\begin{array}{l}1.2296 \times 10^{-7} \pm 1.6088 \times 10^{-} \\
912\end{array}$ \\
\hline & & 8 & $\begin{array}{c}399 \pm \\
7^{7}\end{array}$ & $\begin{array}{c}1.673^{ \pm} \\
0.031^{11,12} \\
\end{array}$ & $\begin{array}{c}1.67 \pm \\
0.031^{11,12} \\
\end{array}$ & 0.997 & $\begin{array}{l}2.3761 \times 10^{-7} \pm 4.45646 \times \\
10^{-916}\end{array}$ \\
\hline
\end{tabular}

${ }^{1-16}$ Different numbers in superscript in Table $1-3$ indicate the statistically significant difference between values, at a significance level of $p<0.05$

Table 4. Activation energy for thin layer dehydration of potato slices.

\begin{tabular}{|c|c|c|c|c|c|c|c|c|c|}
\hline $\begin{array}{c}\mathrm{T} \\
\left({ }^{\circ} \mathrm{C}\right)\end{array}$ & $\begin{array}{c}\mathbf{m} \\
\left(\mathrm{kg} \mathrm{m}^{-2}\right)\end{array}$ & $\begin{array}{c}\mathrm{d} \\
(\mathrm{mm})\end{array}$ & $\begin{array}{c}E_{\mathrm{a}} \\
\left(\mathrm{kJ} \mathrm{mol}^{-1}\right)\end{array}$ & $\underset{\left(\mathrm{kg} \mathrm{m}^{-2}\right)}{\mathbf{m}}$ & $\begin{array}{c}\text { d } \\
(\mathrm{mm})\end{array}$ & $\begin{array}{c}\mathrm{E}_{\mathrm{a}} \\
\left(\mathrm{kJ} \mathrm{mol}^{-1}\right)\end{array}$ & $\underset{\left(\mathrm{kg} \mathrm{m}^{-2}\right)}{\mathbf{m}}$ & $\begin{array}{c}d \\
(\mathbf{m m})\end{array}$ & $\begin{array}{c}\mathbf{E}_{\mathrm{a}} \\
\left(\mathrm{kJ} \mathrm{mol}^{-1}\right)\end{array}$ \\
\hline 35 & \multirow{3}{*}{1.00} & 3 & 24.12 & \multirow{3}{*}{0.63} & 3 & 25.93 & \multirow{3}{*}{0.38} & 3 & 27.02 \\
\hline 50 & & 5 & 22.44 & & 5 & 23.51 & & 5 & 24.56 \\
\hline 70 & & 8 & 21.03 & & 8 & 22.88 & & 8 & 23.25 \\
\hline
\end{tabular}

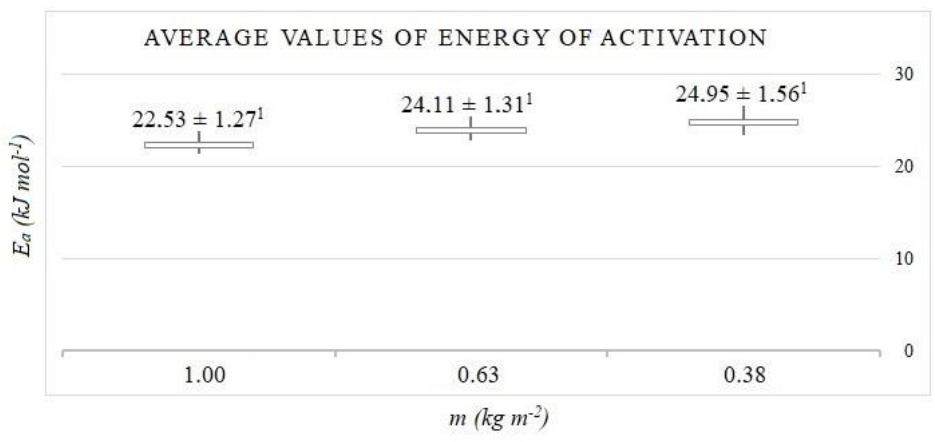

Fig. 1. The average values and standard deviations of activation energy at drying temperatures ( 35 , $\left.50,70^{\circ} \mathrm{C}\right)$ and thickness $(3,5,8 \mathrm{~mm})$ of potato slices of thin layer convective dehydration.

Different numbers in superscript in Figure 1 indicate the statistically significant difference between values, at a significance level of $p<0.05$ 
Table 5. Analysis of variance of dehydration time, energy consumption, the effective moisture diffusivity, and activation energy of potato slices of thin layer convective dehydration.

\begin{tabular}{|c|c|c|c|c|c|c|}
\hline \multirow{2}{*}{\multicolumn{2}{|c|}{ Term }} & \multirow{2}{*}{$\mathbf{d f ^ { 1 }}$} & \multicolumn{4}{|c|}{ Sum of squares } \\
\hline & & & $t$ & $E$ & $D_{\text {eff }}$ & $E_{a}$ \\
\hline \multirow{2}{*}{ Temperature } & Linear & 1 & $1343697^{*}$ & $1.133^{*}$ & $9.365 \times 10^{-8 *}$ & $47.968^{*}$ \\
\hline & Quadratic & 1 & $211101^{*}$ & $3.160^{*}$ & $7.672 \times 10^{-8 *}$ & $3.548 *$ \\
\hline \multirow{2}{*}{ Thickness } & Linear & 1 & 618680* & $4,867^{*}$ & $9.377 \times 10^{-8} *$ & $3.749 \times 10^{-32}$ \\
\hline & Quadratic & 1 & 41735 & 0.065 & $8.514 \times 10^{-8}$ & $3.901 \times 10^{-66}$ \\
\hline \multirow[t]{2}{*}{ Mass load } & Linear & 1 & $566623^{*}$ & $3.113^{*}$ & $-3.344 \times 10^{-8 *}$ & $25.687 *$ \\
\hline & Quadratic & 1 & 5811 & 0.012 & $-3.168 \times 10^{-8}$ & 0.107 \\
\hline \multicolumn{2}{|l|}{ Cross product } & 1 & 16686.82 & 0.396 & $1.285 \times 10^{-15}$ & 0.048 \\
\hline \multirow{2}{*}{ Error } & $\begin{array}{l}\text { Residual } \\
\text { variance }\end{array}$ & 17 & 283673 & 0.673 & $2.184 \times 10^{-15}$ & 0.819 \\
\hline & $\begin{array}{l}\text { Total sum of } \\
\text { squares }\end{array}$ & 26 & 3319502 & 13.488 & $7.209 \times 10^{-14}$ & 78.939 \\
\hline \multicolumn{3}{|l|}{$\mathrm{R}^{2}$} & 0.914 & 0.950 & 0.969 & 0.989 \\
\hline
\end{tabular}

$*$ = Statistically significant at level of significance of $p<0.05$

$1=\mathrm{df}-$ degrees of freedom

Table 6. Regression coefficients of $S O P$ (RSM) of dehydration time, energy consumption, the effective moisture diffusivity, and activation energy of potato slices of thin layer convective dehydration.

\begin{tabular}{|c|c|c|c|c|}
\hline & $\mathbf{t}$ & $\mathbf{E}$ & $\mathbf{D}_{\text {eff }}$ & $\mathbf{E}_{\mathbf{a}}$ \\
\hline$\beta_{0}$ & 978.219 & $-7.468^{*}$ & $1.883 \times 10^{-7} *$ & $38.208^{*}$ \\
\hline$\beta_{1}$ & $-54.885^{*}$ & $0.275^{*}$ & $6.982 \times 10^{-9} *$ & $-0.382^{*}$ \\
\hline$\beta_{11}$ & $0.627^{*}$ & $-0.002^{*}$ & $5.464 \times 10^{-11 *}$ & $0.003^{*}$ \\
\hline$\beta_{2}$ & 1014.630 & 2.121 & $4.751 \times 10^{-8}$ & -2.232 \\
\hline$\beta_{22}$ & 329.892 & -0.470 & $4.878 \times 10^{-8}$ & -1.451 \\
\hline$\beta_{3}$ & 208.730 & 0.048 & $-1.603 \times 10^{-9}$ & $3.544 \times 10^{-15}$ \\
\hline$\beta_{33}$ & -13.993 & 0.014 & $8.988 \times 10^{-10}$ & $-7,798 \times 10^{-30}$ \\
\hline$\beta_{12}$ & $-29.340^{*}$ & $0.010^{*}$ & $-1.5614 \times 10^{-9} *$ & $0.0260^{*}$ \\
\hline$\beta_{13}$ & -1.148 & $0.001^{*}$ & $3.6724 \times 10^{-10 *}$ & $-5.256 \times 10^{-17}$ \\
\hline$\beta_{23}$ & $115.119^{*}$ & $0.072^{*}$ & $-1.5265 \times 10^{-8 *}$ & $-1.237 \times 10^{-15}$ \\
\hline
\end{tabular}

$*=$ Statistically significant at $p<0.05$ level.

Agricultural products (fruits and vegetables) and food containing high moisture contents react very intensively for dehydration; these products could absorb the energy quickly as long as there was remaining moisture [21]. The obtained experimental results (Tables $1-3$ ) showed that at the same mass load, increasing the dehydration temperature and potato slice thickness, statistically significantly $(\mathrm{p}<0.05)$, increased the energy consumption $(\mathrm{E})$. As far as the mass load was decreased, the energy consumption was decreased too. The smallest energy consumption $(0.176 \mathrm{kWh})$ had the model of potato slices with the $3 \mathrm{~mm}$ thickness, $0.38 \mathrm{~kg} \mathrm{~m}-2$ mass load, dehydrated on temperature $70{ }^{\circ} \mathrm{C}$ (as well as the dehydration time). Unlike the dehydration time, the highest energy consumption values had the models dehydrated at a temperature $50{ }^{\circ} \mathrm{C}$; increasing the thickness of potato slices and mass load, the energy consumption increased. The highest energy consumption had a model of potato dehydration on $50{ }^{\circ} \mathrm{C}, 8 \mathrm{~mm}$ thickness, and a $1.00 \mathrm{~kg} \mathrm{~m}-2$ mass load. The total energy was increased as the potato slice thickness was increased [22]. Since the mathematical correlation between energy consumption and carbon dioxide emission has already been mentioned, $\mathrm{CO} 2$ emission monitors energy consumption. Parameter CO2/E in Tables $1-3$ shows the experimental mathematical correlation of dehydrated models (the declared value of the Prosto manufacturer was 0.998 ). 
The calculated average values for effective moisture diffusivity (Deff) for the different dehydrated models of potato slices are presented in Tables $1-3$, and the calculated values of the activation energy (Ea) are presented in Table 4 and Figure 1.

By increasing the dehydration temperature and the potato slice thickness and decreasing the mass load, the values for effective moisture diffusivity were statistically significantly $(\mathrm{p}<0.05)$ increased. These results are also in correlation with reports by Chijijoke et al. [22]. This means that potato slices of $8 \mathrm{~mm}$ slice thickness dried at $70{ }^{\circ} \mathrm{C}$, with $0.38 \mathrm{~kg} \mathrm{~m}-2$ mass load, showed the highest resistance to mass transfer (the maximum Deff $=2.3761 \times 10-7 \pm$ $4.45646 \times 10-9 \mathrm{~m} 2 \mathrm{~s}-1)$.

The activation energy $(\mathrm{Ea})$ was statistically significantly $(\mathrm{p}<0.05)$ decreased with the increasing potato slice thickness (Table $4 \&$ Figure 1). For the dehydration models, lower activation energy means more effective moisture diffusivity (higher coefficient of mass transfer) and an increasing the moisture diffusion with slice thickness that breaking of the bond between the water molecules of the sample is occurred by lower energy consumption [3]. Increasing the dehydration temperature will definitely decrease the activation energy, unlike mass load. The minimum activation energy was obtained for the dehydration of potato slices on $70^{\circ} \mathrm{C}, 8 \mathrm{~mm}$ thickness and $1.00 \mathrm{~kg} \mathrm{~m}-2$ mass load $(\mathrm{Ea}=27.02 \mathrm{~kJ}$ mol-1), unlike the maximum activation energy on $35^{\circ} \mathrm{C}, 3 \mathrm{~mm}$ thickness and $0.38 \mathrm{~kg} \mathrm{~m}-2$ mass load $(\mathrm{Ea}=$ $21.03 \mathrm{~kJ}$ mol-1). Decreasing the mass load (Figure 1), the activation energy was increased, with the maximum activation energy Ea, $0.38 \mathrm{~kg} \mathrm{~m}-2=24.95 \pm 1.56 \mathrm{~kJ}$ mol-1. The range of calculated activation energy correlated with the general range of activation energy [22].

The results showed that both linear and quadratic terms of dehydration temperature for all tested responses ( $t, E$, Deff, Ea) were statistically significant $(p<0.05)$. Unlike, the linear terms of dehydration mass load and potato slice thickness were statistically significant. High values of R2 (from 0.914 to 0.989 ) for all dehydration models indicated a good prediction of developed models for the experimental data.

The regression coefficients for all SOP dehydration models of dehydration time, energy consumption, the effective moisture diffusivity, and activation energy are shown in Table 6. The statistical significance of individual coefficients was noted. Data from Table 6 could be used to complete SOP quadratic equations of potato slice dehydration and the dehydration process's prediction and

Generally, a strong correlation was noticed for all dehydration parameters (temperature, potato slice thickness, mass load), of which the temperature and the potato slice thickness stand out as the dominant factor for defining the optimal dehydration time, the optimal energy consumption, and the emission of carbon dioxide, and effective moisture diffusivity. The dominant factors for defining the optimal activation energy was the temperature and the mass load.

\section{Conclusions}

The dehydration time, energy consumption, carbon dioxide emission, effective moisture diffusivity, and activation energy of potato slice dehydration statistically significantly depend on convective hot air dehydration parameters (temperature, potato slice thickness, and mass load).

The short dehydration time (183 minutes), the smallest energy consumption $(0.176 \mathrm{kWh})$, and the smallest emission of carbon dioxide $(0.17 \mathrm{~kg})$ had the dehydration model of potato slices with the $3 \mathrm{~mm}$ thickness, $0.38 \mathrm{~kg} \mathrm{~m}-2$ mass load, dehydrated on the temperature of 70 ${ }^{\circ} \mathrm{C}$. Increasing the dehydration temperature and potato slice thickness, the dehydration time, the energy consumption, and carbon dioxide emission were increased. As far as the mass load was increased for these dehydration models, the dehydration time, energy consumption, and carbon dioxide emission were increased. 
By increasing the dehydration temperature and the potato slice thickness, and decreasing the mass load, the effective moisture diffusivity was increased, and the activation energy was decreased. Dehydration of potato slices of $8 \mathrm{~mm}$ slice thickness dehydrated at $70{ }^{\circ} \mathrm{C}$, with $0.38 \mathrm{~kg} \mathrm{~m}-2$ mass load, showed the highest resistance to mass transfer (the maximum effective moisture diffusivity $2.3761 \times 10-7 \pm 4.45646 \times 10-9 \mathrm{~m} 2 \mathrm{~s}-1)$. The same dehydration model showed the minimum activation energy (27.02 $\mathrm{kJ}$ mol-1), unlike the maximum activation energy for dehydration models obtained on $35^{\circ} \mathrm{C}, 3 \mathrm{~mm}$ thickness and $0.38 \mathrm{~kg} \mathrm{~m}$ 2 mass load (21.03 kJ mol-1). Decreasing the mass load, the effective moisture diffusivity was decreased, and the activation energy was increased.

Developed mathematical models of potato slice dehydration showed a good correlation between measured and calculated results, allowing the creation of a database to optimize the dehydration process.

\section{Acknowledgments}

The authors would like to acknowledge the Don State Technical University for supporting publishing this study financially.

\section{References}

1. C.R. Amiri, N. Dibagar, and B. Alaei, Heat Mass Transf. 53, 3473-3485 (2017)

2. H. Darvishi et al., J. Agr. Sci. Tech. 20, 249-264 (2018)

3. H. Azimi-Nejadian, and S.S. Hoseini, Heat Mass Transf. 55, 2921-2930 (2019)

4. V. Filipović et al., IOP Conf. Ser.: Mater. Sci. Eng. 900, 012001 (2020)

5. M.C. Ndukwu, et al., Case Stud. Therm. Eng. 9, 62-71 (2017)

6. E.A. Kosasih, A. Zikri, and M.I Dzaky, Case Stud. Therm. Eng. 19, 100633 (2020)

7. T. Defraeye, and P. Verboven, J. Food Eng. 193, 95-107 (2017)

8. X.L. Zhang et al., J. Food Eng. 241, 51-57 (2019)

9. L.S. Montoya-Ballesteros et al., Dry. Technol. 32, 1486-1499 (2014)

10. B. Moshen, Food Sci. Technol. 36, 145-150 (2016)

11. V. Chuwattanakul, and S. Eiamsaard, Case Stud. Therm. Eng. 13, 100389 (2019)

12. M. Beigi, Chem. Ind. Chem. Eng. Q. 23, 431-440 (2017)

13. M. Petković et al., Period. Polytech. Chem. Eng. 63, 600-608 (2019)

14. M Petković et al., IOP Conf. Ser.: Mater. Sci. Eng. 1029, 012046 (2021)

15. Z. Erbay, and F. Icier, Crit. Rev. Food Sci. Nutr. 50, 441-464 (2010)

16. M. Petković et al., J. Food Process. Preserv. 45, e15027 (2020)

17. Prosto PM 001 Manual (2020) https://www.elementa.rs/files/52018-a.pdf

18. Statistica StatSoft Inc. 2013 Data analysis software system (Version 12), https://statistica.software.informer.com/12.0/

19. N. Hafezi, M.J. Sheikhdavoodi, and S.M. Sajadiye, Int. J. Agric. Food Res., 424-431 (2015)

20. D.I. Onwude et al., Heat Mass Transf. 54, 2917-2933 (2018)

21. Ö. Süfer, S. Sezar, and H. Demir, J. Food Process Preserv. 41, e13239 (2017)

22. Ch.E. Onu, Ph.K. Igbokwe, J.T. Nwabanne, Int. J. Novel Res. Eng. Sci. 3, 10-22 (2016) 